Revue internationale P.M.E.

Économie et gestion de la petite et moyenne entreprise

\title{
La structure du capital des petites et moyennes entreprises industrielles tunisiennes
}

\section{Mohamed Ali Omri, Moncef Akremi et Meryem Bellouma}

Volume 18, numéro 2, 2005

URI : https://id.erudit.org/iderudit/1008478ar

DOI : https://doi.org/10.7202/1008478ar

Aller au sommaire du numéro

Éditeur(s)

Presses de l’Université du Québec

ISSN

0776-5436 (imprimé)

1918-9699 (numérique)

Découvrir la revue

Citer cette note

Ali Omri, M., Akremi, M. \& Bellouma, M. (2005). La structure du capital des petites et moyennes entreprises industrielles tunisiennes. Revue internationale P.M.E., 18(2), 141-163. https://doi.org/10.7202/1008478ar
Résumé de l'article

L'objectif de cet article est d'étudier la pertinence des différentes théories de financement dans l'explication du choix de la structure du capital au sein des petites et moyennes entreprises industrielles tunisiennes (PMI). Nous avons effectué une analyse empirique sur $300 \mathrm{PMI}$ tunisiennes durant la période allant de 1997 à 2003. Nos résultats montrent que la décision de financement dans ces entreprises pourrait être expliquée par les principales théories de structure du capital (théorie fiscale, théorie de l'endettement optimal et théorie du financement hiérarchique). La théorie du financement hiérarchique semble le mieux convenir pour expliquer la politique de financement des PMI. 


\title{
La structure du capital des petites et moyennes entreprises industrielles tunisiennes
}

Mohamed ALI OMRI

Faculté des sciences économiques et de gestion de Tunis

Moncef AKREMI

Faculté des sciences économiques et de gestion de Tunis

Meryem BELLOUMA

Institut supérieur de gestion de Tunis

\section{MOTS CLÉS}

\section{Petites et moyennes entreprises - Structure du capital Entreprises tunisiennes}

\begin{abstract}
RÉSUMÉ
L'objectif de cet article est d'étudier la pertinence des différentes théories de financement dans l'explication du choix de la structure du capital au sein des petites et moyennes entreprises industrielles tunisiennes (PMI). Nous avons effectué une analyse empirique sur 300 PMI tunisiennes durant la période allant de 1997 à
\end{abstract}

\section{LES AUTEURS}

MoHAmed ALI OMRI est maître-assistant, habilité en sciences de gestion à la Faculté des sciences économiques et de gestion de Tunis et directeur de recherche à l'unité de recherche en finance et stratégie des affaires (FIESTA). Auteur de plusieurs articles sur la fiscalité et la gouvernance d'entreprise, ses principaux intérêts de recherche concernent la fiscalité, la performance, ainsi que la gestion des résultats des entreprises. Adresse: Faculté des sciences économiques et de gestion de Tunis, B.P. 248, Manar II, Tunisie. Courriel: <medali@yahoo.fr>

MONCEF AKREMI est doctorant chercheur en gestion à la Faculté des sciences économiques et de gestion de Tunis et membre de l'unité de recherche FIESTA. Ses recherches portent sur le domaine fiscal et la performance des entreprises.

MERYEM BELLOUMA est doctorante chercheure à l'Institut supérieur de gestion de Tunis et membre de l'unité de recherche FIESTA. Ses recherches portent sur le domaine bancaire et sur les relations contractuelles de crédit; elle s'intéresse également à la performance et à la gouvernance d'entreprise. Courriel <mbellouma@yahoo.fr>. 
2003. Nos résultats montrent que la décision de financement dans ces entreprises pourrait être expliquée par les principales théories de structure du capital (théorie fiscale, théorie de l'endettement optimal et théorie du financement hiérarchique). La théorie du financement hiérarchique semble le mieux convenir pour expliquer la politique de financement des PMI.

\section{ABSTRACT}

The principal aim of this paper is to test the relevance of the different financing theories used to explain the capital structure of the Tunisian small and medium enterprises (SME). To establish the main determinants of their capital structure, our study is based on a sample of 300 SME, over a period from 1997 to 2003. Our results show that the financing decision in these companies could be explained by the main capital structure theories: fiscal theory, trade-off theory and pecking order theory. Among all these theories, the pecking order theory seems to fit in the explanation of SMEs debt policy.

\section{RESUMEN}

La puntería principal de este papel es probar la importancia de las diversas teorías del financiamiento usadas para explicar la estructura de capital de las empresas pequeñas y medias tunecinas (PyMEs). Para establecer los determinantes principales de su estructura de capital, nuestro estudio se basa en una muestra de 300 PyMEs, sobre un período 1997-2003. Nuestros resultados demuestran que la decisión del financiamiento en estas compañías se podría explicar por la teoría principal de la estructura de capital: teoría fiscal, teoría de la compensación y picotear teoría de la orden. Entre todas estas teorías, la teoría de la orden que picotea se parece caber en la explicación de la política de la deuda de PyMEs.

\section{ZUSAMMENFASSUNG}

Die Zielsetzung dieses Artikels besteht darin, die Aussagekraft verschiedener Finanzierungstheorien bei der Wahl der Struktur des Kapitals von kleinen und mittleren tunesischen Industrieunternehmen zu untersuchen. Wir haben eine empirische Untersuchung während 1997 bis 2003 bei 300 besagten tunesischen Unternehmen durchgeführt. Die erhaltenen Resultate zeigen auf, dass die Entscheidung über die Art der Finanzierung in diesen Unternehmen mit den gängigsten Finanzierungstheorien (Steuer-Theorie, Theorie der optimalen Verschuldung und Theorie der hierarchischen Finanzierung) erklärt werden kann. Die Theorie der hierarchischen Finanzierung scheint sich am besten zur Erklärung der Finanzierungspolitik zu eignen.

Unsere Ergebnisse zeigen, dass die Finanzierungsentscheidungen in diesen Unternehmen durch die wesentlichen Theorien der Kapitalstruktur erklärt werden könnte (fiskalische Theorie, Theorie der optimalen Verschuldung und hierarchische Finanzierungstheorie). Die hierarchische Finanzierungstheorie ist am besten geeignet, um die Finanzierungspolitik der kleinen und mittleren Industrieunternehmen zu erklären. 


\section{Introduction}

Les petites et moyennes entreprises constituent un segment prépondérant de l'économie tunisienne en tant que source majeure de vitalité, de souplesse et surtout de création d'emplois. Cependant, il n'existe aucun consensus sur la définition ${ }^{1}$ de l'expression «petites et moyennes entreprises (PME)». En effet, des concepts divers (chiffre d'affaires annuel, nombre d'employés, forme juridique, etc.) ont fréquemment été utilisés pour définir les spécificités de la $\mathrm{PME}^{2}$. Le rapport Bolton (1971), qui fut à l'origine de la reconsidération des PME par l'économie industrielle en Angleterre, retient deux critères essentiellement qualitatifs. Une PME est une entreprise qui détient une part relativement petite de son marché et qui est gérée de manière personnelle par son propriétaire qui prend des décisions sans contrôle extérieur.

Dans le cadre de cet article, nous nous concentrerons sur le problème du financement des petites et moyennes entreprises. Cette orientation est justifiée par les nombreuses décisions des pouvoirs publics ainsi que par les modalités de création et d'expansion des PME. En effet, cette catégorie d'entreprises, entachée de fragilité et d'opacité informationnelle, fait face à des taux d'intérêts prohibitifs ${ }^{3}$ ou à un rationnement de crédit en raison de son incapacité à fournir les garanties nécessaires (Ang, 1991; Black et Strahan, 2002; Bodt, Lobez et Statnik, 2001).

Par ailleurs, les problèmes de financement varient considérablement entre les entreprises déjà établies et les nouvelles entreprises, ainsi qu'entre celles qui se développent lentement et celles qui croissent rapidement. D'où la nécessité

1. Sauf autres précisions, une petite et moyenne entreprise (PME) est une entité économique ayant l'une des formes de sociétés telles qu'elles sont définies par le code des sociétés commerciales, entré en vigueur depuis 2000 et un effectif d'employés ne dépassant pas 100 personnes en moyenne sur l'année.

2. En Tunisie, aucune définition officielle de la PME n'est avancée, ni par les pouvoirs publics, ni par aucune autre organisation ou institution. Cependant, dans le cadre de sa mission de vérification approfondie des dossiers fiscaux, l'administration fiscale procède à l'étude des dossiers, sur différents aspects, des différentes personnes physiques et morales avant de les programmer. L'un des critères retenus est le chiffre d'affaires des contribuables. Selon ce critère, les petites et moyennes entreprises sont celles ayant un chiffre d'affaires inférieur à 5 millions de dinars (en 2003, en moyenne: 1 dollar américain équivaut 1,3 dinar tunisien et 1 dollar canadien équivaut 0,9 dinar tunisien).

3. Les taux d'intérêt font l'objet de négociations entre la banque et l'entreprise. L'existence de garanties réelles et la participation de la banque dans le capital de la société sont parmi les termes essentiels de toute négociation portant sur le montant du crédit, la période de remboursement et les taux d'intérêts appliqués.

Revue internationale P.M.E., vol. 18, nº 2, 2005

(c) 2005 - Presses de l'Université du Québec

Édifice Le Delta I, 2875, boul. Laurier, bureau 450, Sainte-Foy, Québec G1V 2M2 - Tél.: (418) 657-4399 - www.puq.ca

Tiré de: Revue internationale P.M.E., vol. 18, $\mathrm{n}^{\circ} 2$, sous la direction de Louis Raymond • PME1802N

Tous droits de reproduction, de traduction et d'adaptation réservés 
d'esquisser les principaux déterminants du financement des PME tunisiennes, à la lumière des théories afférentes à la structure de capital. Notre étude sera centrée sur les petites et moyennes entreprises industrielles tunisiennes, désormais nommées PMI.

L'expansion des marchés privés de fonds propres et, notamment, les nouvelles mesures à caractère juridique ont sensiblement amélioré l'accès au capital-risque pour les nouvelles entreprises et les PMI, mais des différences sensibles subsistent entre les pays. En Tunisie, par exemple, depuis 2001, une loi est consacrée aux modalités de financement par le capital-risque. Par ailleurs, à partir de 2000, les PMI peuvent être constituées sous la forme d'entreprise unipersonnelle, forme qui permet au capital de ces entreprises d'avoir une certaine stabilité, généralement exigée pour leur financement externe.

Depuis les travaux fondamentaux de Modigliani et Miller (1958), de nombreuses recherches, théoriques et empiriques, se sont intéressées à l'étude de la structure financière des entreprises (Harris et Raviv, 1991). Cependant, la question de leur applicabilité et de leur pertinence pour le cas d'une petite ou moyenne entreprise demeure, toutefois, sous étude (Belletante et Levratto, 1995).

En effet, les études empiriques sur la structure du capital et ses dynamiques ont principalement traité des grandes entreprises (Anderson et Fraser, 20004; Zingales, 2000). Les premières études empiriques ont porté sur les décisions de financement des entreprises cotées (Bradlay, Jarrell et Kim, 1984; Auberbach, 1985). Plusieurs chercheurs se sont intéressés aux petites et moyennes entreprises (Constand, Osteryoung et Nast, 1991; Van der Wijst, 1989; Walker, 1989; Holmes et Kent, 1991; Van der Wijst et Thurik, 1993; Chittenden, Hall et Hutchinson, 1996; Jordan, Lowe et Taylor, 1998; Michaela, Chittenden et Poutziouris, 1999). Dans la lignée de ces travaux, notre étude vise à soumettre une réflexion sur la décision de la PMI en matière de financement. Notre recherche permettra d'établir les déterminants de la politique de financement des petites et moyennes entreprises industrielles tunisiennes, et tentera de répondre à la question suivante: dans quelle mesure les principales théories de la structure du capital peuvent expliquer les décisions relatives au financement des PMI tunisiennes?

Pour ce faire, nous recenserons, en premier lieu (section 1), les fondements théoriques de la structure du capital. Nous essayerons, en deuxième lieu (section 2) de cerner le degré de validité de ces théories en ce qui concerne l'identification des déterminants de la politique de financement de la PMI tunisienne, en matière de choix de la structure de financement. Finalement, nous présenterons les principales conclusions et limites de l'étude (conclusion).

4. L'étude empirique de ces deux auteurs n'a concerné que les banques.

Revue internationale P.M.E., vol. 18, nº 2, 2005

(C) 2005 - Presses de l'Université du Québec

Édifice Le Delta I, 2875, boul. Laurier, bureau 450, Sainte-Foy, Québec G1V 2M2 - Tél.: (418) 657-4399 - www.puq.ca

Tiré de: Revue internationale P.M.E., vol. 18, $\mathrm{n}^{\circ} 2$, sous la direction de Louis Raymond PME1802N

Tous droits de reproduction, de traduction et d'adaptation réservés 


\section{Fondements théoriques de la structure du capital}

Modigliani et Miller (1958) ont été les premiers à analyser l'incidence de la structure financière et notamment du ratio dettes/fonds propres sur la valeur de la firme. Ils montrent ainsi que, sous certaines conditions, toutes les formes de financement sont équivalentes en présence de marchés financiers parfaits.

La sélection d'une structure financière quelconque repose principalement sur les conditions restrictives suivantes:

- les marchés financiers sont parfaits;

- les agents peuvent prêter ou emprunter à un taux fixe et sans limite;

- les coûts de faillite sont nuls ;

- il n'y a pas d'asymétrie d'information entre les agents ;

- les dirigeants gèrent leur entreprise dans l'intérêt de leurs actionnaires (absence de conflits d'intérêts).

La remise en cause progressive de ces conditions extrêmement limitatives a permis de faire ressortir l'influence de plusieurs facteurs déterminants sur la structure financière, ce qui a permis de rejeter l'hypothèse de neutralité de la structure de financement. Dans le cadre de cette section, nous essayerons de cerner l'effet de l'impôt, de la dette et du financement hiérarchique sur la structure du capital.

\subsection{Influence de la fiscalité sur la structure du capital}

En essayant de se rapprocher davantage de la réalité de l'entreprise, Modigliani et Miller (1963) ont pris en compte l'influence de la fiscalité et, en particulier, la déductibilité des charges financières sur le résultat imposable. Cette orientation a remis en cause la thèse de la neutralité des choix de financement sur la valeur de l'entreprise, qui s'avère être plus élevée pour une entreprise endettée que pour une entreprise totalement financée par des fonds propres. Après avoir tenu compte du rôle de la fiscalité, on constate donc que les entreprises préfèrent l'endettement à d'autres sources de financement, puisqu'elles peuvent bénéficier de la déductibilité des intérêts de la dette de l'assiette soumise à l'impôt.

Pour le financement des PMI, Pettit et Singer (1985) considèrent que la dette n'est pas appropriée à leur structure fragile et qu'elle est sujette à une probabilité de défaillances relativement élevées par rapport aux grandes entreprises. Ainsi, les gains d'impôts procurés par l'endettement peuvent s'estomper par les coûts de

Revue internationale P.M.E., vol. 18, n 2, 2005

(C) 2005 - Presses de l'Université du Québec

Édifice Le Delta I, 2875, boul. Laurier, bureau 450, Sainte-Foy, Québec G1V 2M2 • Tél.: (418) 657-4399 - www.puq.ca

Tiré de: Revue internationale P.M.E., vol. 18, n 2, sous la direction de Louis Raymond • PME1802N

Tous droits de reproduction, de traduction et d'adaptation réservés 
défaillance qui peuvent en découler (Warner, 1977; Smith et Warner, 1979; Ang, Chua et McConnell, 1982). Cette évidence est appuyée par la théorie de l'endettement optimal de la structure du capital. En effet, le financement par dettes ne présente pas uniquement des avantages, mais aussi des imperfections matérialisées par le risque financier (coût de faillite) et les coûts d'agence qui émergent entre créanciers et dirigeants. De ce fait, Pettit et Singer (1985) affirment que la théorie fiscale ne peut pas être appliquée dans le contexte des PMI, surtout que leur relation avec les bailleurs de fonds est entachée d'asymétrie d'information.

En revanche, certains auteurs (Auerbach, 2002; Graham et Rogers, 2002) ont mis l'accent sur le report des pertes et des amortissements différés ${ }^{5}$, autorisés par la plupart des législations en vigueur, pour justifier l'applicabilité de la théorie fiscale aussi bien pour les entreprises déficitaires que pour les bénéficiaires. Par ailleurs, le fait de ne pas soumettre l'opération d'augmentation de capital ${ }^{6}$ à l'impôt encouragera le recours à un meilleur financement externe.

En Tunisie, le taux d'imposition des sociétés de $35 \%$ est calculé sur le bénéfice comptable corrigé (bénéfice fiscal). Les charges d'intérêts sont déductibles de l'assiette imposable, mais les dividendes sont exonérés chez la personne, physique ou morale, qui les reçoit. Par ailleurs, le législateur a réservé à l'opération d'augmentation de capital un traitement qui le distingue ${ }^{7}$ des autres opérations de transfert soumises à l'impôt.

La théorie fiscale est tour à tour attaquée sur différents aspects, notamment celui des gains d'impôt résultant de la dette. L'une des principales critiques adressées à cette théorie rappelle l'existence, en pratique, d'une fiscalité hétérogène entre les individus et les entreprises et les incidences des coûts de faillite sur le choix d'une structure financière optimale.

Il semble, par conséquent, que la théorie fiscale soit incapable, à elle seule, d'expliquer les choix de financement des entreprises tunisiennes. D'autres théories sont nécessaires pour une meilleure intelligibilité du cas tunisien.

5. En Tunisie, par exemple, les pertes d'exploitation sont reportables sur quatre ans. Les pertes résultant d'amortissements différés peuvent être reportées indéfiniment.

6. Le législateur fiscal tunisien, par exemple, distingue nettement entre les opérations de transferts de biens assimilées à des ventes, donc imposables et les opérations d'augmentation de capital, définies comme étant des apports en sociétés effectuées en franchise d'impôt.

7. Les participations à l'augmentation de capital des entreprises ne sont pas imposables à l'impôt sur les sociétés.

Revue internationale P.M.E., vol. 18, nº 2, 2005

(C) 2005 - Presses de l'Université du Québec

Édifice Le Delta I, 2875, boul. Laurier, bureau 450, Sainte-Foy, Québec G1V 2M2 - Tél.: (418) 657-4399 - www.puq.ca

Tiré de: Revue internationale P.M.E., vol. 18, $\mathrm{n}^{\circ} 2$, sous la direction de Louis Raymond PME1802N

Tous droits de reproduction, de traduction et d'adaptation réservés 


\subsection{Théorie de l'endettement optimal}

La théorie de l'agence, résultante de la remise en cause de l'hypothèse d'absence de conflits entre les acteurs de l'entreprise, étudie les conflits d'intérêts qui peuvent survenir, d'une part, entre les actionnaires et les créanciers et, d'autre part, entre les actionnaires et les dirigeants.

En effet, le bailleur de fonds peut être sujet, en raison de l'asymétrie d'information, au détournement de la part de l'entreprise d'une partie des gains liés à l'investissement (Williamson, 1986). Afin de limiter l'apparition de ce phénomène, le créancier introduit des clauses restrictives dans le contrat de la dette, notamment pour les échéances. Ce qui se traduit par l'écartement des PMI du champ du crédit à moyen et long terme, relativement aux grandes firmes. Ainsi, l'asymétrie d'information peut expliquer en partie la structure de la dette de ces entreprises.

En plus de cette alternative, les créanciers peuvent exiger de la PMI l'apport de garanties dont le volume et la nature sont spécifiés de manière à inciter l'entreprise à respecter ses engagements. Pour Besanko et Thakor (1987), l'apport de garanties peut ainsi limiter le risque moral de l'entreprise (ou du dirigeant), du fait qu'elle se trouve menacée d'une liquidation potentielle de son actif ${ }^{8}$, si elle ne peut respecter ses engagements.

Ainsi, dans ces conditions, la structure de capital optimale peut être obtenue par arbitrage entre les avantages de l'endettement (la déductibilité des charges financières) et les coûts d'agence relatifs aux relations avec les créanciers, qui peuvent emmener l'entreprise, passant par une période de détresse financière, à supporter des coûts de faillite.

La prise en compte de la fiscalité, du risque de défaillance et des conflits d'intérêts entre les différents agents participant à la vie de l'entreprise a donné lieu à une analyse de la structure de capital de l'entreprise, qui se démarque de celle de la théorie du financement optimal, à savoir l'approche de financement hiérarchique «pecking order». Nous synthétisons cette approche en rapport avec l'objet de notre étude, la PMI.

8. Selon Barclay et Smith (1999), quand les entreprises avec des opportunités élevées de croissance utilisent le financement par crédit, elles préfèrent les dettes à court terme ou les dettes avec des restrictions faibles à des dettes à long terme afin d'avoir plus de flexibilité.

Revue internationale P.M.E., vol. 18, n 2, 2005

(c) 2005 - Presses de l'Université du Québec

Édifice Le Delta I, 2875, boul. Laurier, bureau 450, Sainte-Foy, Québec G1V 2M2 - Tél.: (418) 657-4399 - www.puq.ca

Tiré de: Revue internationale P.M.E., vol. 18, $\mathrm{n}^{\circ} 2$, sous la direction de Louis Raymond P PME1802N

Tous droits de reproduction, de traduction et d'adaptation réservés 


\subsection{Théorie du financement hiérarchique}

En raison des asymétries d'information entre les différents acteurs de la vie économique et financière de l'entreprise, les dirigeants préfèrent les sources de financement internes par rapport aux sources de financement externes ${ }^{9}$. Cela suggère que les entreprises à forte rentabilité tendent à autofinancer leurs investissements (par les excédents de bénéfices non distribués) plutôt que de recourir à la dette. Ainsi, les dirigeants des PMI, ne voulant pas perdre la propriété et le contrôle de leurs entreprises, dont ils sont généralement les actionnaires, préfèrent épuiser leurs fonds propres avant de contracter des dettes ou procéder à une augmentation de capital. D'où la notion du financement hiérarchique avancée par Myers (1984) et Myers et Majluf (1984). Dans le cas où les PMI auraient besoin de sources de financement externes, elles choisiront le type d'endettement qui ne réduit pas les prérogatives des dirigeants. En l'occurrence, elles opteront pour l'endettement à court terme qui ne contient pas normalement des engagements restrictifs.

En conclusion, la théorie du financement hiérarchique revient à affirmer l'inséparabilité entre les décisions d'investissement et de financement. En ce qui concerne les petites ou moyennes entreprises, comme l'ont soutenu Myers et Majluf (1984), deux variables interviennent dans le classement des modes de financement. Ces deux variables sont les préférences du dirigeant et les possibilités d'accéder au marché financier. En plus, les banques considèrent que les petites ou moyennes entreprises ont un risque élevé de faillite. Par conséquent, elles se méfient beaucoup de leur accorder des emprunts (Belletante, 1996).

L'évolution de la théorie financière, mettant l'accent sur la fiscalité, les coûts de faillite et l'asymétrie d'information, a rejeté l'idée que le niveau d'endettement n'aurait aucune influence sur la valeur de la firme. Ainsi, le choix entre le financement externe et le financement interne s'effectue en fonction de l'arbitrage lié à l'importance du risque de défaut de l'entreprise. D'où l'émergence de plusieurs hiérarchies de financement en fonction du critère de maximisation du dirigeant (pecking order). Tous ces développements montrent que le problème du choix du financement des activités de l'entreprise, et particulièrement de la PMI, est assez

9. En matière de financement externe, le choix est plutôt porté sur la dette peu risquée (celle qui présente beaucoup de garanties et qui nécessite peu d'efforts pour convaincre les créanciers). Ensuite, le choix porte sur les dettes plus risquées (c'est-à-dire les dettes dont la probabilité de ne pas être remboursées dans leur intégralité n'est pas nulle et les titres hybrides par émission d'obligations convertibles). Enfin, c'est l'augmentation de capital à laquelle les entreprises n'auront recours que lorsque leur taux d'endettement ne permet pas d'emprunter davantage. Cette émission d'actions engendre un signal négatif qu'il faudrait limiter en réduisant l'asymétrie de l'information (Myers et Majluf, 1984).

Revue internationale P.M.E., vol. 18, n 2, 2005

(C) 2005 - Presses de l'Université du Québec

Édifice Le Delta I, 2875, boul. Laurier, bureau 450, Sainte-Foy, Québec G1V 2M2 - Tél.: (418) 657-4399 - www.puq.ca

Tiré de: Revue internationale P.M.E., vol. 18, n², sous la direction de Louis Raymond • PME1802N

Tous droits de reproduction, de traduction et d'adaptation réservés 
complexe. Après avoir présenté les bases théoriques sur lesquelles s'appuient les recherches sur la structure de capital des entreprises, nous tentons, dans ce qui suit, de mettre en évidence l'existence de certaines variables explicatives ainsi que les relations et les prédictions présumées auparavant.

\section{2. Étude empirique des déterminants de la structure du capital des PMI tunisiennes}

L'objectif de cette recherche est de connaître les déterminants de la structure du capital des petites et moyennes entreprises industrielles tunisiennes. Pour ce faire, nous avons considéré un échantillon de 300 petites et moyennes entreprises tunisiennes appartenant au secteur industriel. Toutes les entreprises retenues dans l'échantillon ont satisfait aux critères suivants ${ }^{10}$ :

- un effectif d'employés par PMI inférieur à 100;

- le chiffre d'affaires ne dépasse pas les 5 millions de dinars tunisiens (TND), soit environ 3,846 millions de dollars américains.

Les données ont été collectées à partir des bilans comptables et des notes aux états financiers des entreprises sur une période de sept ans (1997-2003) ${ }^{11}$. Nos sources de données sont variées : à part les données recueillies auprès de l'Institut national des statistiques (INS), nous avons complété et confronté ces données à celles issues du ministère des Finances et du ministère de l'Industrie (Agence de promotion des industries, API).

Le choix de la période (1997-2003) s'explique, d'une part, par la mise en place en 1997 du nouveau système comptable des entreprises. D'autre part, cette période coïncide avec le démarrage du $9^{\mathrm{e}}$ plan de développement économique et social de la Tunisie, qui explicite les politiques et les stratégies gouvernementales à entreprendre pour la direction du pays. Les années 2002 et 2003 sont présentées par les pouvoirs publics comme étant des années de renforcement des acquis du $9^{e}$ plan de développement économique et social et du démarrage du $10^{\mathrm{e}}$ plan. Ce dernier plan a pour ambition de «gagner le défi de l'emploi, asseoir l'économie

10. Comme nous l'avons signalé plus haut, ce choix est justifié aussi bien au plan de l'effectif des employés retenu qu'à celui du chiffre d'affaires. En ce qui concerne l'effectif, nous avons repris celui retenu par Van der Wijst (1989). En ce qui concerne le chiffre d'affaires, c'est le seuil à partir duquel l'administration fiscale tunisienne considère que l'affaire n'est plus une petite ou moyenne entreprise.

11. Ces données n'ont subi aucun retraitement. Par conséquent, tous les agrégats suivants sont comptables.

Revue internationale P.M.E., vol. 18, n 2, 2005

(C) 2005 - Presses de l'Université du Québec

Édifice Le Delta I, 2875, boul. Laurier, bureau 450, Sainte-Foy, Québec G1V 2M2 - Tél.: (418) 657-4399 - www.puq.ca

Tiré de: Revue internationale P.M.E., vol. 18, n², sous la direction de Louis Raymond • PME1802N

Tous droits de reproduction, de traduction et d'adaptation réservés 
du savoir et consolider la compétitivité ${ }^{12} \gg$. De tels objectifs sont généralement associés au succès des PME (Nejadan, 1997; Favre, 2003). En effet, celles-ci, à cause de leur poids économique et de leur rôle déterminant sur la croissance, sont productrices d'une forte valeur ajoutée et potentiellement créatrices d'emplois (Favre, 2003).

En vue d'assurer une meilleure représentativité de l'échantillon, nous avons opté pour la méthode des quotas. Dans le choix de notre échantillon, nous avons essayé de reproduire certaines caractéristiques de la population des petites et moyennes entreprises tunisiennes.

Les tableaux suivants donnent les principales caractéristiques des entreprises choisies. Le premier tableau présente la répartition des entreprises par secteur d'activité. Le test d'homogénéité a permis de retenir l'hypothèse nulle selon laquelle les deux populations (totale et échantillon) ont une répartition homogène entre les six secteurs d'activité au seuil de $5 \%$.

TABLEAU 1

\section{Répartition des petites et moyennes entreprises} par secteur d'activité et test d'homogénéité

\begin{tabular}{|c|c|c|c|c|}
\hline \multirow[b]{2}{*}{ Secteur d'activité } & \multicolumn{2}{|l|}{ Population } & \multicolumn{2}{|l|}{ Échantillon } \\
\hline & $\begin{array}{l}\text { Nombre } \\
\text { d'entreprises* }\end{array}$ & Pourcentage & $\begin{array}{l}\text { Nombre } \\
\text { d'entreprises }\end{array}$ & $\begin{array}{l}\text { Effectifs } \\
\text { théoriques** }\end{array}$ \\
\hline $\begin{array}{l}\text { Industrie agricole } \\
\text { et alimentaire }\end{array}$ & 1260 & 16,75 & 54 & 50,26 \\
\hline $\begin{array}{l}\text { Industrie des matériaux } \\
\text { de construction }\end{array}$ & 929 & 12,35 & 41 & 37,06 \\
\hline $\begin{array}{l}\text { Industrie mécanique } \\
\text { et électrique }\end{array}$ & 801 & 10,65 & 34 & 31,95 \\
\hline Industrie chimique & 720 & 9,57 & 28 & 28,72 \\
\hline Industrie textile et cuir & 2890 & 38,43 & 102 & 115,29 \\
\hline Industries diverses & 920 & 12,23 & 41 & 36,70 \\
\hline Total & 7520 & 100 & 300 & 300 \\
\hline Test d'homogénéité & \multicolumn{4}{|c|}{$\begin{array}{l}Q \text { observé }=2,88 ; \chi(\text { avec degré de liberté égal à } 5)=11,07 \\
\text { au seuil de } 5 \%\end{array}$} \\
\hline
\end{tabular}

* Le nombre d'entreprises est une moyenne sur les sept dernières années.

** Effectif théorique $=$ taille échantillon $\times$ pourcentage (industrie agricole et alimentaire $300 \times 16,75 \%=50,26)$.

Source: Données de l'API (Agence de promotion des industries), INS (Institut national des statistiques) et ministère des Finances.

12. Citation tirée du $10^{\mathrm{e}}$ plan de développement économique et social de la Tunisie (2002-2006) 
Le deuxième tableau recense les entreprises selon leur taille. Le test d'homogénéité montre que l'échantillon est représentatif au seuil de $5 \%$.

TABLEAU 2

Répartition des entreprises par taille et test d'homogénéité

\begin{tabular}{|c|c|c|c|c|c|c|c|c|}
\hline & \multicolumn{8}{|c|}{ Classes de chiffres d'affaires (en million de dinars tunisien) } \\
\hline & {$[0-0,2]$} & {$[0,2-0,5]$} & {$[0,5-1,5]$} & {$[1,5-3]$} & {$[3-3,8]$} & {$[3,8-4,3]$} & {$[4,3-5]$} & Total \\
\hline Population & 639 & 987 & 690 & 1543 & 1519 & 823 & 1319 & 7520 \\
\hline Pourcentage & 8,49 & 13,12 & 9,17 & 20,51 & 20,2 & 10,94 & 17,53 & 100 \\
\hline Échantillon & 30 & 45 & 35 & 44 & 53 & 38 & 55 & 300 \\
\hline $\begin{array}{l}\text { Effectifs } \\
\text { théoriques }\end{array}$ & 25,49 & 39,37 & 27,52 & 61,55 & 60,59 & 32,83 & 52,62 & 300 \\
\hline $\begin{array}{l}\text { Test } \\
\text { d'homogénéité }\end{array}$ & \multicolumn{8}{|c|}{$\begin{array}{l}Q \text { observé }=10,51 ; \chi(\text { avec degré de liberté égal à } 6)=12,59 \\
\text { au seuil de } 5 \%\end{array}$} \\
\hline
\end{tabular}

Source: Données de l'API (Agence de promotion des industries), INS (Institut national des statistiques) et ministère des Finances.

Ainsi, la synthèse des données reprises ci-dessus confirme que l'échantillon est représentatif au seuil de $5 \%$.

Toutes les entreprises retenues dans l'échantillon possèdent les caractéristiques suivantes:

- détiennent un total d'actifs inférieur ou égal à 10 millions de dinars (environ 7,7 millions de dollars américains);

- ont réalisé des bénéfices tout au long de la période d'étude et ne sont pas en difficultés financières;

- ne sont pas exonérées de l'impôt sur les sociétés, ni situées hors champs;

- ne sont pas des filiales de grandes sociétés (y compris les banques);

- n'ont pas effectué des augmentations de capital par incorporation de réserves provenant de réinvestissements exonérés ${ }^{13}$.

13. Le code des avantages fiscaux promulgués depuis 1993 prévoit une multitude d'encouragements au profit de l'entreprise. Certains instruments visent le renforcement de la capitalisation des entreprises. L'un de ces instruments est l'exonération des réinvestissements physiques et financiers.

Revue internationale P.M.E., vol. 18, nº 2, 2005

(C) 2005 - Presses de l'Université du Québec

Édifice Le Delta I, 2875, boul. Laurier, bureau 450, Sainte-Foy, Québec G1V 2M2 - Tél.: (418) 657-4399 - www.puq.ca

Tiré de: Revue internationale P.M.E., vol. 18, n² , sous la direction de Louis Raymond • PME1802N

Tous droits de reproduction, de traduction et d'adaptation réservés 


\subsection{Variables retenues dans l'analyse}

Dans cette sous-section, nous présenterons la variable à expliquer et les variables explicatives.

\subsubsection{Variable à expliquer: endettement des PMI}

La variable à expliquer par le modèle est le ratio d'endettement des PMI. Cette variable est mesurée par le rapport suivant: dettes brutes totales ${ }^{14} /$ actif total. L'actif total est constitué de l'actif non courant et de l'actif courant qui sont extraits directement des bilans comptables des petites ou moyennes entreprises de l'échantillon. Cependant, l'analyse des causes déterminantes de la structure du capital basée seulement sur la dette brute totale peut révéler des différences importantes entre la dette à long terme et la dette à court terme (Van der Wijst et Thurik, 1993; Chittenden, Hall et Hutchinson, 1996; Barclay et Smith, 1999; Bevan et Danbolt, 2000). En conséquence, pour mieux comprendre la structure du capital et ses déterminants, nous considérons les mesures de levier suivantes:

- $\mathbf{R D T T}_{i, t}=$ ratio de la dette totale. Cette variable sera mesurée par le rapport dettes totales/actif total;

- $\mathbf{R D L T}_{i, t}=$ ratio de la dette à long terme. Cette variable sera mesurée par le rapport dettes à long terme/actif total;

- $\mathbf{R D C T}_{i, t}=$ ratio de la dette à court terme. Cette variable sera mesurée par le rapport dettes à court terme/actif total.

\subsubsection{Variables explicatives}

Les variables explicatives de la structure du capital proposées ont été appréhendées par un ensemble de critères utilisés dans la littérature empirique (Titman et Wessels, 1988; Harris et Raviv, 1991).

\section{A. Fiscalité}

La variable fiscale (TXIE) est mesurée par le taux effectif moyen d'imposition des sociétés/ (bénéfices après charges financières et avant impôts). Sur le plan empirique, Titman et Wessels (1988) n'ont pas réussi à démontrer que la variable

14. Dans ce qui suit, nous simplifierons cette expression par «dettes totales».

Revue internationale P.M.E., vol. 18, nº 2, 2005

(C) 2005 - Presses de l'Université du Québec

Édifice Le Delta I, 2875, boul. Laurier, bureau 450, Sainte-Foy, Québec G1V 2M2 • Tél.: (418) 657-4399 - www.puq.ca

Tiré de: Revue internationale P.M.E., vol. 18, $\mathrm{n}^{\circ} 2$, sous la direction de Louis Raymond PME1802N

Tous droits de reproduction, de traduction et d'adaptation réservés 
fiscale a un effet significatif sur l'endettement. Talmorn, Haugen et Senbet (1986) ont relevé l'existence d'une relation positive entre la variable fiscale et l'endettement pour les entreprises qui réalisent des bénéfices imposables importants. Contrairement aux prédictions de Talmorn, Haugen et Senbet (1986), Kremp, Stöss et Gerdesmeier (1999) supposent l'existence d'une relation négative entre le taux d'imposition et le niveau d'endettement.

Ces prédictions et résultats controversés indiquent que la problématique de l'existence ou non et le sens de la relation (si elle existe) entre la fiscalité et l'endettement reste entière pour les PMI. Dans le cadre de notre recherche, nous nous attendons à ce que la relation entre la variable fiscale et l'endettement soit positive, surtout que toutes les entreprises de l'échantillon sont bénéficiaires ${ }^{15}$.

\section{B. Économie d'impôt non liée à la dette}

Selon Modigliani et Miller (1958), la déduction fiscale des charges financières a un effet positif sur l'accroissement de l'endettement. En revanche, pour les PMI dont le revenu imposable est faible, l'incitation à l'endettement est modérée par l'existence d'autres charges déductibles, comme les amortissements et les provisions. Puisque notre échantillon est essentiellement composé d'entreprises exerçant dans des secteurs d'activité où l'actif amortissable est utilisé de façon intensive, nous avons choisi de mesurer les économies d'impôt non liées à la dette (EIND) par le rapport des charges non décaissées (dotations aux amortissements et aux provisions)/actif brut total. En Tunisie, la loi fiscale admet l'amortissement accéléré du matériel affecté à l'actif de l'entreprise, lorsque celle-ci justifie son utilisation par plus d'une équipe. Cette loi autorise aussi la pratique de l'amortissement dégressif. Ces deux modes d'amortissement ont pour effet d'accélérer le remplacement de l'actif amortissable en place. Cela a pour effet d'augmenter les économies d'impôt pour les entreprises ayant un actif remplaçable important. Dans le cadre de notre recherche, nous nous attendons à ce que les économies de l'impôt non liées à la dette soient négativement corrélées avec l'effet de levier financier.

15. Malgré le fait que même les entreprises déficitaires ont intérêt à utiliser la variable fiscale pour reporter les pertes, d'une part, et participer aux programmes de sauvetage, prévus par les autorités compétentes (Ministère des Finances et ministère de l'Industrie), des sociétés en difficultés, d'autre part.

Revue internationale P.M.E., vol. 18, n 2, 2005

(C) 2005 - Presses de l'Université du Québec

Édifice Le Delta I, 2875, boul. Laurier, bureau 450, Sainte-Foy, Québec G1V 2M2 • Tél.: (418) 657-4399 - www.puq.ca

Tiré de: Revue internationale P.M.E., vol. 18, n², sous la direction de Louis Raymond • PME1802N

Tous droits de reproduction, de traduction et d'adaptation réservés 


\section{Opportunités de croissance}

Les études empiriques utilisent généralement le $\mathrm{Q}$ de Tobin pour mesurer les opportunités de croissance (De Jong et Veld, 2000). Cependant, comme le Q de Tobin n'est pas observable, certaines approximations de cette mesure ont été proposées par les chercheurs (Perfect et Wiles, 1994). Wanzenried (2002) a proposé, comme indicateur des opportunités de croissance, la variation relative de l'actif total d'une année à l'autre. Antoniou, Guney et Paudyal (2002) ont défini les opportunités de croissance en se référant à la valeur de marché de l'actif. L'étude des bilans comptables et des notes aux états financiers ${ }^{16}$ des entreprises de l'échantillon indique que leurs actifs se composent essentiellement de l'outil de production. Dans notre recherche basée sur des données comptables, nous ne mesurons que les opportunités de croissance connues. Ainsi, nous appréhendons l'existence pour l'entreprise d'opportunités de croissance (OPCR) à l'aide de la variable taux de renouvellement des immobilisations, qui est mesurée par le ratio (variation des immobilisations nettes ${ }^{17}+$ dotation aux amortissements)/dotations aux amortissements. Ce ratio vise à déterminer l'importance des dépenses d'investissement. Il révèle un développement de l'outil de production (ratio supérieur à 1) ou un vieillissement de l'équipement productif (ratio inférieur à 1). Il permet aussi d'apprécier la capacité de l'entreprise à saisir des opportunités de croissance et peut donc signaler un risque d'investissement sous-optimal (Jensen et Meckling, 1976; Jensen, 1986).

Les développements de la théorie de financement hiérarchique prévoient que les opportunités de croissance sont positivement corrélées avec l'effet de levier financier de l'entreprise (Micheala, Chittenden et Poutziouris, 1999; Kremp, Stöss et Gerdesmeier, 1999).

Les relations attendues dans le cadre de notre recherche sont les suivantes:

- les dettes à long terme sont négativement corrélées avec les opportunités de croissance;

- les dettes à court terme ont une corrélation positive avec les opportunités de croissance.

16. Les notes aux états financiers expliquent le contenu du bilan et des autres états financiers.

17. On suppose toutefois que les éventuelles cessions d'actifs se font sur la base des valeurs nettes comptables.

Revue internationale P.M.E., vol. 18, nº 2, 2005

(C) 2005 - Presses de l'Université du Québec

Édifice Le Delta I, 2875, boul. Laurier, bureau 450, Sainte-Foy, Québec G1V 2M2 - Tél.: (418) 657-4399 - www.puq.ca

Tiré de: Revue internationale P.M.E., vol. 18, $\mathrm{n}^{\circ} 2$, sous la direction de Louis Raymond P PME1802N

Tous droits de reproduction, de traduction et d'adaptation réservés 


\section{Structure de l'actif}

La structure de l'actif (SACT) renseigne sur la capacité de l'entreprise d'offrir des garanties. Elle est mesurée par le rapport suivant: montant des immobilisations corporelles/actif brut total.

Les entreprises ayant d'importants actifs immobilisés peuvent bénéficier de conditions d'endettement plus favorables. Dans une situation d'asymétrie d'information, il est facile pour les prêteurs d'évaluer la valeur des actifs matériels comparés aux actifs immatériels (Wanzenried, 2002; Antoniou, Guney et Paudyal, 2002). De même, il existe une forte probabilité que la valeur des actifs immatériels diminue radicalement en cas de difficultés financières ou de faillite. Nous nous attendons à ce que les dettes totales soient corrélées positivement avec la tangibilité des actifs.

Selon le principe d'assortiment de maturité de Brealey et Myers (2000), l'hypothèse précédente a été élargie dans le sens suivant: dans le cas où les sociétés visent à assortir les maturités des actifs et des passifs, nous devrions observer une corrélation positive entre le ratio des immobilisations et le ratio des dettes si elles sont contractées à long terme.

Nous escomptons, toutefois, que le ratio des immobilisations et le ratio des dettes à court terme soient négativement corrélés.

\section{E. Rentabilité}

Dans le cadre de la théorie du financement hiérarchique, Titman et Wessels (1988) proposent de considérer la rentabilité (RENT) comme un indicateur de la disponibilité des financements internes. La rentabilité économique représente la rentabilité de l'ensemble des capitaux engagés dans l'entreprise. Elle donne une mesure de ce que rapporte tout le capital engagé pour un endettement nul et hors considérations fiscales. Elle est mesurée par le ratio: résultat courant avant intérêt et impôt/actif économique. Ce ratio est déterminé avant impôt et intérêts de façon à ne dépendre ni de la politique de financement, ni de la pression fiscale. L'ensemble des moyens mis en œuvre est constitué des capitaux propres et des dettes financières.

Nous pensons, par ailleurs, que les PMI utilisent principalement les dettes à court terme comme moyen de financement.

Revue internationale P.M.E., vol. 18, nº 2, 2005

(c) 2005 - Presses de l'Université du Québec

Édifice Le Delta I, 2875, boul. Laurier, bureau 450, Sainte-Foy, Québec G1V 2M2 • Tél.: (418) 657-4399 - www.puq.ca

Tiré de: Revue internationale P.M.E., vol. 18, $\mathrm{n}^{\circ} 2$, sous la direction de Louis Raymond PME1802N

Tous droits de reproduction, de traduction et d'adaptation réservés 


\subsection{Méthodologie}

Pour mesurer l'influence des différentes variables explicatives sur la structure du capital, nous avons utilisé les techniques économétriques d'estimation sur données de panel incomplet ${ }^{18}$. L'indice $i$ représente l'entreprise et l'indice $t$, la période considérée. Le modèle à estimer se présente comme suit:

$$
\mathrm{Y}_{i, t}=\mathrm{X}_{i, t} * \beta+\alpha_{i}+\mu_{i, t}
$$

Où $\mathrm{Y}_{i, t}$ représente la variable endogène de mesure de l'endettement retenue pour l'entreprise $i$ à l'année $t ; \mathrm{X}$ est le vecteur des variables explicatives retenues ; $\beta$ est le vecteur des coefficients; $\alpha_{i}$ représente l'effet spécifique à l'entreprise $i$ supposé être fixe ou aléatoire et $\mu_{i, t}$ est un terme d'erreurs résiduelles.

\subsection{Résultats empiriques}

\subsubsection{Analyse descriptive}

Les statistiques descriptives des variables expliquées et explicatives retenues sont présentées au tableau 3.

TABLEAU 3

Statistiques descriptives

\begin{tabular}{lcccc}
\hline Variables & Moyenne & Écart type & Minimum & Maximum \\
\hline RDTT & 0,5833 & 0,2521 & 0 & 0,9827 \\
RDLT & 0,0852 & 0,1678 & 0 & 0,9187 \\
RDCT & 0,4732 & 0,2721 & 0 & 0,9830 \\
TXIE & 0,1913 & 0,1457 & 0 & 0,8834 \\
EIND & 0,0423 & 0,0512 & 0 & 0,8182 \\
OPCR & 1,2731 & 0,0931 & 0,8515 & 5,2731 \\
SACT & 0,4728 & 0,2721 & 0 & 1 \\
RENT & 0,0951 & 0,0933 & $-1,584$ & 3,4352 \\
\hline
\end{tabular}

18. Pour vérifier le caractère des effets individuels, le test de spécification de Hausman (1978) est habituellement utilisé. Il s'agit de vérifier la validité de l'hypothèse nulle selon laquelle les effets individuels ne sont pas corrélés avec les variables indépendantes. Si nous acceptons l'hypothèse nulle (H0), les effets individuels sont censés être aléatoires et nous devons appliquer la méthode des moindres carrés généralisés à notre modèle avec des estimateurs de variables instrumentaux. Cependant, si nous rejetons $\mathrm{H} 0$, alors les effets individuels sont fixes et l'estimateur des moindres carrés généralisés devient biaisé et non consistant. Dans ce dernier cas, nous devons utiliser l'estimateur Within qui est identique à l'estimateur des moindres carrés ordinaires obtenu à partir d'un modèle transformé où les variables expliquées et explicatives sont centrées sur leurs moyennes individuelles respectives.

Revue internationale P.M.E., vol. 18, n 2, 2005

(C) 2005 - Presses de I'Université du Québec

Édifice Le Delta I, 2875, boul. Laurier, bureau 450, Sainte-Foy, Québec G1V 2M2 • Tél.: (418) 657-4399 - www.puq.ca

Tiré de: Revue internationale P.M.E., vol. 18, n², sous la direction de Louis Raymond • PME1802N

Tous droits de reproduction, de traduction et d'adaptation réservés 
Pour examiner le degré de corrélation possible entre les variables, nous avons obtenu la matrice de corrélation des variables dépendantes et indépendantes qui se présente comme suit (tableau 4).

TABLEAU 4

Matrice des corrélations

\begin{tabular}{|c|c|c|c|c|c|c|c|c|}
\hline Variables & $\begin{array}{c}1- \\
\text { RDTT }\end{array}$ & $\begin{array}{c}2- \\
\text { RDLT }\end{array}$ & $\begin{array}{c}3- \\
\text { RDCT }\end{array}$ & $\begin{array}{c}4- \\
\text { TXIE } \\
\end{array}$ & $\begin{array}{c}5- \\
\text { EIND }\end{array}$ & $\begin{array}{c}6- \\
\text { OPCR }\end{array}$ & $\begin{array}{c}7- \\
\text { SACT }\end{array}$ & $\begin{array}{c}\text { 8- } \\
\text { RENT }\end{array}$ \\
\hline 1 - RDTT & 1 & & & & & & & \\
\hline $2-\mathrm{RDLT}$ & $0,22 *$ & 1 & & & & & & \\
\hline $3-\mathrm{RDCT}$ & $0,35 *$ & $-0,24 *$ & 1 & & & & & \\
\hline $4-\mathrm{TXIE}$ & $-0,17 * *$ & $-0,18 * *$ & $-0,04 *$ & 1 & & & & \\
\hline 5 - EIND & $-0,15^{*}$ & $0,12^{*}$ & $-0,18 * *$ & $-0,33 *$ & 1 & & & \\
\hline $6-\mathrm{OPCR}$ & $0,14 * *$ & $0,25^{*}$ & $-0,05^{*}$ & $-0,14 *$ & $0,27 * *$ & 1 & & \\
\hline $7-\mathrm{SACT}$ & $0,04 *$ & $0,22 *$ & $-0,13 *$ & $-0,22 *$ & $0,12 *$ & $-0,15^{*}$ & 1 & \\
\hline 8 - RENT & $-0,13 *$ & $-0,28 * *$ & $-0,12 *$ & $0,28 *$ & $0,03 *$ & $0,03 * *$ & $-0,12 * *$ & 1 \\
\hline
\end{tabular}

* Significatif à $5 \%$.

** Significatif à $10 \%$.

La matrice des corrélations présente, d'une part, la relation estimée entre les différentes variables explicatives et, d'autre part, la relation prévue entre la variable dépendante (endettement) et les variables indépendantes. Cette matrice montre que le degré de corrélation entre les différentes variables indépendantes est modéré; ce qui suppose l'absence du problème de multicolinéarité entre les variables.

\subsubsection{Résultats des régressions}

Les résultats de l'estimation à partir des données de panel incomplet indiquent que la prise en compte de la spécificité individuelle des entreprises sous forme d'un effet fixe donne de meilleurs résultats sur le plan de la significativité statistique en comparaison d'un modèle à effet individuel aléatoire.

Nos résultats montrent que toutes les variables sont statistiquement significatives et que le test $\mathrm{F}$ souligne le besoin de considérer toutes les variables d'un point de vue statistique.

Par ailleurs, le taux d'impôt effectif semble avoir une relation négative significative avec l'effet de levier de l'entreprise. Cela peut être interprété comme révélant l'existence d'une relation inverse de cause à effet entre les impôts et les dettes de l'entreprise. Dans ce cas, les entreprises avec des niveaux d'endettement élevés payeraient moins d'impôts. Il semble, par conséquent, que les dirigeants

Revue internationale P.M.E., vol. 18, n 2, 2005

(C) 2005 - Presses de l'Université du Québec

Édifice Le Delta I, 2875, boul. Laurier, bureau 450, Sainte-Foy, Québec G1V 2M2 - Tél.: (418) 657-4399 - www.puq.ca

Tiré de: Revue internationale P.M.E., vol. 18, n², sous la direction de Louis Raymond • PME1802N

Tous droits de reproduction, de traduction et d'adaptation réservés 


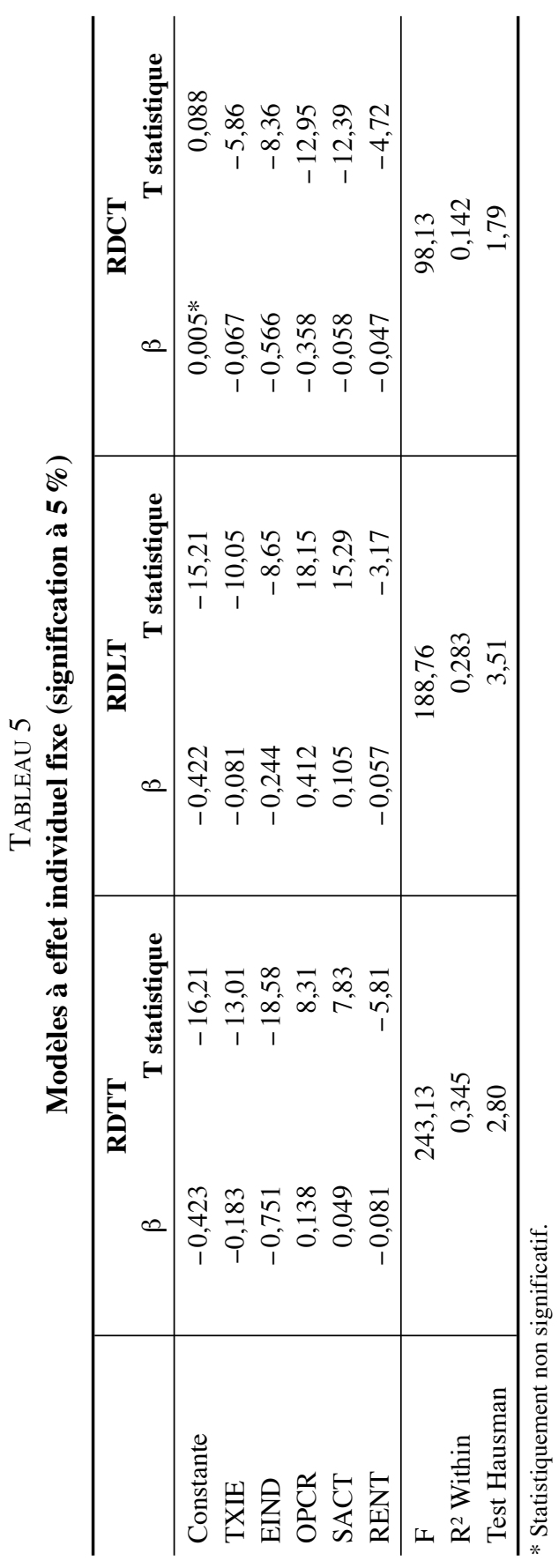

Revue internationale P.M.E., vol. 18, nº 2, 2005 
des PMI ne recourent pas à l'endettement pour réduire leur engagement fiscal, mais utilisent d'autres dispositifs pour ce faire. Les économies d'impôt non liées à la dette semblent être négativement liées avec les dettes.

De même, les PMI qui possèdent des opportunités de croissance élevées ont plus de dettes dans leur structure du capital. Néanmoins, une corrélation négative significative apparaît entre le taux de renouvellement des investissements et les dettes à court terme; ce qui traduit les différentes durées de vie de ces types d'actif et de passif.

En ce qui concerne la variable structure de l'actif, elle est positivement liée à l'effet de levier total de l'entreprise. Ce résultat conforte les prédictions de la théorie de l'agence. Selon cette théorie, la capacité de l'entreprise à offrir des garanties réduit d'autant les coûts d'agence et incite davantage les créanciers à s'engager dans des prêts à longues échéances (Jensen et Meckling, 1976; Harris et Raviv, 1991). L'existence de garanties est un critère de sélection pour les offres de prêts des banques. Plus l'entreprise offre de garanties, moins elle sera contrainte dans son accès au financement bancaire.

La corrélation entre l'effet de levier et la structure de l'actif change de manière significative selon le type de ratio d'effet de levier utilisé. Particulièrement, nous constatons que le ratio de dettes à long terme est positivement corrélé avec la structure d'actifs, alors que cette corrélation devient négative en présence du ratio de dettes à court terme. Le même résultat est obtenu par Van der Wijst (1989), Van der Wijst et Thurik (1993), Chittenden, Hall et Hutchinson (1996) et Van der Wijst (1997). La variable structure d'actifs mesure le ratio d'actifs corporels rapportés aux actifs totaux. Les actifs corporels sont constitués principalement par les immobilisations, qui sont retenues durablement dans l'actif. La corrélation négative entre la structure d'actifs et le ratio de dettes à court terme signifie que les dettes à court terme (passifs courants) sont utilisées pour financer les actifs non immobilisés, principaux constituants des actifs courants. C'est ce qui a déjà été appelé «principe d'assortiment de maturité » par Brealey et Myers (2000).

Enfin, le signe négatif de la rentabilité est l'une des évidences de la théorie du financement hiérarchique de Myers (1984) et Myers et Majluf (1984). Selon cette théorie, les PMI les plus profitables ont moins tendance à recourir à l'endettement pour financer leur activité.

\section{Conclusion}

L'objectif de l'article est de cerner la politique financière des PMI tunisiennes, à la lumière de la théorie fiscale, de la théorie de l'endettement optimal et de celle du financement hiérarchique.

Revue internationale P.M.E., vol. 18, n 2, 2005

(C) 2005 - Presses de l'Université du Québec

Édifice Le Delta I, 2875, boul. Laurier, bureau 450, Sainte-Foy, Québec G1V 2M2 - Tél.: (418) 657-4399 - www.puq.ca

Tiré de: Revue internationale P.M.E., vol. 18, n², sous la direction de Louis Raymond • PME1802N

Tous droits de reproduction, de traduction et d'adaptation réservés 
Dans le cadre de la revue de la littérature, nous avons présenté l'évolution de la théorie relative aux déterminants de la structure de capital des entreprises, à la suite de la remise en question progressive des hypothèses de base du modèle de Modigliani et Miller (1958). Nous avons pu souligner l'importance des coûts d'agence liés à l'asymétrie d'information et le rôle de la fiscalité sur la structure du financement et la valeur de la petite et moyenne entreprise. Nous avons aussi mis en évidence l'importance des théories qui défendent les modes de financement hiérarchisés dans l'explication des politiques financières des PMI.

Afin d'éclaircir le choix de financement des PMI tunisiennes et d'expliciter leur approche dans le choix du niveau d'endettement, nous avons utilisé un panel composé de 300 PMI sur la période s'étalant de 1997 à 2003.

En ce qui concerne la théorie fiscale, nous constatons que l'effet de levier est sensiblement corrélé avec les économies d'impôts non liées à la dette telles que l'amortissement et les provisions. La structure des actifs présente une corrélation positive avec le niveau des dettes à long terme et une corrélation négative avec le niveau des dettes à court terme. Cela peut démontrer le principe d'assortiment de maturité dans les PMI. Les dirigeants cherchent à financer les immobilisations par des dettes à long terme et les actifs courants par des dettes à court terme.

En outre, les PMI ayant des opportunités de croissance importantes semblent davantage recourir à l'endettement, bien que ce rapport soit négatif lorsqu'il s'agit d'utiliser les dettes à court terme. Ce fait peut suggérer que ce genre d'actifs est lié à sa nature caractérisée par un horizon long et ainsi son financement devrait l'assortir. Nous remarquons aussi que les PMI s'appuient, en priorité, pour leur financement sur les ressources internes au détriment des ressources externes de la société.

En conclusion, la théorie de financement hiérarchique semble bien expliquer la politique d'endettement des PMI tunisiennes.

Toutefois, cette étude pourrait être poussée plus loin et considérer une période d'analyse plus étendue afin de vérifier l'existence d'un changement cyclique dans la structure du capital des PMI. D'autres études pourraient porter uniquement sur les PMI qui réalisent des résultats déficitaires. Il serait aussi intéressant de reproduire ce type d'analyse avec des PMI ayant diverses caractéristiques organisationnelles afin d'établir les bases d'une typologie de leur politique en matière de structure de financement. 


\section{Bibliographie}

ANDERSON, R.C. et D.R. FrASER (2000), «Corporate control, bank risk taking and health of the banking industry », Journal of Banking and Finance, vol. 24, $\mathrm{n}^{\circ}$ 8, p. 13831398.

ANG, J.S. (1991), «Small business uniqueness and theory of financial management», Journal of Small Business Finance, vol. 1, $\mathrm{n}^{\mathrm{o}}$ 1, p. 1-13.

ANG, J.S., J.H. ChuA et J.J. McConnell (1982), «The administrative costs of corporate bankruptcy: a note», Journal of Finance, vol. 37, n ${ }^{\circ}$ 1, p. 219-226.

Antoniou, A., Y. Guney et K. Paudyal (2002), «Determinants of corporate capital structure: evidence from European countries », Cahier de recherche, Université de Durham, p. 23-26.

Auerbach, A.J. (1985), «Real determinants of corporate leverage», dans B. Friedman (dir.), Corporate Capital Structures in the United States, Presses de l'Université de Chicago, p. 301-322.

Auerbach, A.J. (2002), «Taxation and corporate financial policy», dans A.J. Auerbach et M. Feldstein (dir.), Handbook of Public Economics, New York, North-Holland, p. 1251-1292.

Barclay, M. J. et C.W. SMith (1999), «The capital structure puzzle: another look the evidence », Journal of Applied Corporate Finance, vol. 12, n ${ }^{\circ}$ 1, p. 8-20.

Belletante, B. (1996), «Les politiques financières des PME cotées sur les marchés financiers », Actes du III Congrès international francophone sur la PME, TroisRivières, p. 10-15.

Belletante, B. et N. Levratto (1995), «Finance et PME: quels champs pour quels enjeux? » Revue internationale PME, vol. 8, nos 3-4, p. 60-65.

BEsAnKo, D. et A.V. THAKOR (1987), «Competitive equilibriumin in the credit market under asymmetric information », Journal of Economic Theory, vol. 42, n 4, p. 167-182.

Bevan, A.A. et J. Danbolt (2000), «Capital structure and its determinants in the United Kingdom: a decompositional analysis », Cahier de recherche 2000-2, Université de Glasgow, p. 69-76.

Black, S.E. et P.E. STRAHAN (2002), «Entrepreneurship and bank credit availability», Journal of Finance, vol. 57, p. 2807-2833.

BodT, E., F. Lobez et J.-C. STATNIK (2001), «Credit rationing, customer relationship and the number of banks: an empirical analysis », Cahier de recherche, Université de Lille II, p. 33-61.

Bolton, J.E. (1971), Small Firms: Report of the Committee on Inquiry on Small Firms, Norwich, R.-U., HMSO, vol. 1, p. 33-48.

Bradlay, M., G.A. JARRELl et E.H. KIM (1984), «On the existence of an optimal capital structure: theory and evidence », Journal of Finance, vol. 39, n 3, p. 857-878.

Brealey, R. A. et S.C. Myers (2000), Principles of Corporate Finance, 6e édition, Blacklick, Ohio, McGraw-Hill, p. 69-102.

Revue internationale P.M.E., vol. 18, nº 2, 2005

(C) 2005 - Presses de l'Université du Québec

Édifice Le Delta I, 2875, boul. Laurier, bureau 450, Sainte-Foy, Québec G1V 2M2 • Tél.: (418) 657-4399 - www.puq.ca

Tiré de: Revue internationale P.M.E., vol. 18, n², sous la direction de Louis Raymond • PME1802N

Tous droits de reproduction, de traduction et d'adaptation réservés 
Chittenden, F.H., G. Hall et P. Hutchinson (1996), « Small firm growth, access to capital markets and financial structure: review of issues and an empirical investigation », Small Business Economics, vol. 8, $\mathrm{n}^{\circ}$ 1, p. 59-67.

Constand, R.L., J.S. Osteryoung et D.A. Nast (1991), «Asset-based financing and the determinants of capital structure in the small firm», dans R. Yazdipour (dir.), Advances in Small Business Finance, Boston, Mass., Kluwer Academic Publishers, p. 29-45.

De Jong, A. et C. Veld (2000), «Share repurchases: international empirical evidence», dans P.W. Moerland (dir.), Economische, institutionele en juridische aspecten van inkoop van eigen aandelen, Deventer, Kluwer Press, p. 55-78.

FAVRE, C. (2003), «Réformer la fiscalité, une nécessité pour les PME», AGEFI, février, p. $20-23$.

Graham, J. et D.A. Rogers (2002), «Do firms hedge in response to tax incentives?», Journal of Finance, vol. 57, n 2, p. 818-819.

Harris, M. et A. RAVIV (1991), «The theory of capital structure», Journal of Finance, vol. 46, $\mathrm{n}^{\circ}$ 1, p. 297-355.

HAUSMAN, J.A. (1978), «Specification tests in econometrics », Econometrica, vol. 46, n 2, p. $1251-1271$.

Holmes, S. et P. KenT (1991), «An empirical analysis of the financial structure of small and large Australian manufacturing enterprises», The Journal of Small Business Finance, vol. 1, no 2, p. 141-154.

JENSEN, M.C. (1986), «Agency costs of free cash flow, corporate finance and take-over», American Economic Review, vol. 76, n 2, p. 323-329.

Jensen, M.C. et W.H. Meckling (1976), «Theory of the firm: managerial behaviour, agency costs and ownership structure», Journal of Financial Economics, vol. 3, no 1 , p. 305-360.

Jordan, J., J. LOWE et P. TAYLOR (1998), «Strategy and financial policy in UK small firms », Journal of Business Finance \& Accounting, vol. 25, n 1, p. 1-27.

KreMP, E., E. StÖsS et D. GERDESMEIER (1999), «Estimation of a debt function: evidence from French and German firm panel data», dans A. Sauve et M. Scheuer (dir.), Corporate Finance in Germany and France, Recherche conjointe de la Deutche Bundesbank et de la Banque de France, p. 140-194.

Michaela, N., F. Chittenden, et P. Poutziouris (1999), «Financial policy and capital structure choice in UK SMEs: empirical evidence from company panel data», Small Business Economics, vol. 12, no 4, p. 113-130.

Modigliani, F. et M.H. Miller (1958), «The cost of capital, corporation finance and the theory of investment», American Economic Review, vol. 68, n 2, p. 261-297.

Modigliani, F. et M.H. Miller (1963), «Corporate income taxes and the cost of capital: a correction », American Economic Review, vol. 2, no 3, p. 433-443.

MYERS, S.C. (1984), «The capital structure puzzle», Journal of Finance, vol. 39, $\mathrm{n}^{\circ} 3$, p. $575-592$.

Revue internationale P.M.E., vol. 18, nº 2, 2005

(C) 2005 - Presses de l'Université du Québec

Édifice Le Delta I, 2875, boul. Laurier, bureau 450, Sainte-Foy, Québec G1V 2M2 • Tél.: (418) 657-4399 - www.puq.ca

Tiré de: Revue internationale P.M.E., vol. 18, $\mathrm{n}^{\circ} 2$, sous la direction de Louis Raymond - PME1802N

Tous droits de reproduction, de traduction et d'adaptation réservés 
MYers, S.C. et N.S. MAJLuF (1984), «Corporate financing and investment decisions when firms have information that investors do not have», Journal of Financial Economics, vol. $13, \mathrm{n}^{\circ} 2$, p. 187-221.

Nejadan, M.Z. (1997), «La fiscalité des PME en Suisse », La Vie économique, no 2.

PERFECT, S.B. et K.W. WILES (1994), «Alternative constructions of Tobin's Q: an empirical comparison », Journal of Empirical Finance, vol. 1, n ${ }^{\text {os }} 3-4$, p. 313-341.

Pettit, R. et R. Singer (1985), «Small business finance: a research agenda», Financial Management, vol. 14, $\mathrm{n}^{\circ}$ 2, p. 47-60.

SMITH, C. et J. WARNER (1979), «On financial contracting: an analysis of bond covenants », Journal of Financial Economics, vol. 7, n 2, p. 117-161.

Talmorn, E., R.A. Haugen et L.W. Senbet (1986), «Debt, dividends and taxes: equilibrium conditions for simultaneous tax neutrality of debt and dividend policies », Research in Finance, vol. 6, no 2, p. 1-27.

Titman, S. et R. Wessels (1988), «The determinants of capital structure choice», Journal of Finance, vol. 43, no 1, p. 1-19.

VAN DER WIJST, N. (1989), «Financial structure in small business. Theory, tests and applications», Lecture Notes in Economics and Mathematical Systems, $\mathrm{n}^{\circ} 320$, Berlin, Springer-Verlag.

VAN DER WIJST, N. (1997), «Firm finance and growth: an empirical analysis », New Operational Approaches for Financial Modelling, vol. 3, n 2, p. 209-220.

VAN DER WiJst, N. et R. THURIK (1993), «Determinants of small firm debt ratios: an analysis of retail panel data », Small Business Economics, vol. 5, ${ }^{\circ}$ 1, p. 55-65.

WALKER, D.A. (1989), «Financing the small firm», Small Business Economics, vol. 1, n 2 , p. $285-296$.

WANZENRIED, G. (2002), «Capital structure dynamics in UK and continental Europe», Cahier de recherche, Université de Californie à Berkeley, p. 33-35.

WARNER, J.B. (1977), «Bankruptcy costs: some evidence», Journal of Finance, vol. 32, $\mathrm{n}^{\circ} 2$, p. 337-347.

Williamson, S. (1986), «Costly monitoring, financial intermediation, and equilibrium credit rationing », Journal of Monetary Economics, vol. 4, nº 2, p. 212-225.

Zingales, L. (2000), «In search of new foundations», Journal of Finance, vol. 55, no 4, p. $1623-1653$.

Revue internationale P.M.E., vol. 18, nº 2, 2005

(C) 2005 - Presses de l'Université du Québec

Édifice Le Delta I, 2875, boul. Laurier, bureau 450, Sainte-Foy, Québec G1V 2M2 - Tél.: (418) 657-4399 - www.puq.ca

Tiré de: Revue internationale P.M.E., vol. 18, n², sous la direction de Louis Raymond・PME1802N

Tous droits de reproduction, de traduction et d'adaptation réservés 
(C) 2005 - Presses de l'Université du Québec

Édifice Le Delta I, 2875, boul. Laurier, bureau 450, Sainte-Foy, Québec G1V 2M2 • Tél.: (418) 657-4399 - www.puq.ca

Tiré de: Revue internationale P.M.E., vol. 18, $\mathrm{n}^{\circ}$ 2, sous la direction de Louis Raymond - PME1802N

Tous droits de reproduction, de traduction et d'adaptation réservés 